\title{
A Research on Supply Chain Integration Strategy Based on Virtual Value Net
}

\author{
Juanqiong Gou, Tingting Ma and Jingjing Li \\ School of Economics and Management, Beijing Jiaotong University, Beijing 100044, P.R. \\ China jq_gou@263.net tingting ma@163.com Jinging@163.com
}

\begin{abstract}
The supply chain is a dynamic and integrated system in which all enterprises are integrated to obtain optimization of the whole chain. Integration is a process of redefining and connecting parts of a whole in order to form a new one. In traditional supply chain integration, the definitions of parts are usually limited by the boundary of the enterprises: the integration emphasizes connecting each enterprise with logistics and information communications.

This paper proposes a theory of a Virtual Value Net in which a supply chain as a whole system consists of three nets: logistics net, information resource net and organization net. The supply chain integration involves how to construct and coordinate these nets and their nodes, so as to provide business value to the final customers, and be adaptable to business and technology changes.

This paper presents a method by which supply chain integration solution is designed, improved and evaluated. The method takes more care about the business processes of the whole supply chain in which the organizations' boundaries are regarded as transparent, activities in business processes are selected and organized as business components, logistics flow and information flow are analyzed and optimized according to the integration degrees of business components. In addition, a comprehensive method on designation, evaluation and improvement of supply chain integration will also be provided.
\end{abstract}

Keywords: Supply chain, Supply chain integration, Virtual value net, Business components, Integration degree

\section{VIRTUAL VALUE NET AND SUPPLY CHAIN INTEGRATION}

The supply chain is a dynamic and integrated system, in which all enterprises are integrated to obtain optimization of the whole chain. The word 'integration' is broadly used in these realms of management and technology. This paper directly cites the definition from $\mathrm{Li}[1]$ that integration is a process by which parts of a whole become more connected so that they are in effect less "part" \& more "whole"; i.e., such that functions formerly carried out by one part are carried out by others, usually vice versa.

The main issue in integration is not how to connect, but how to redefine the parts and their functions. In traditional integration of the supply chain, definitions of the parts of organizations are usually limited by the boundary of the enterprise;

Please use the following format when citing this chapter:

Gou, J., Ma, T., Li, J., 2007, in IFIP International Federation for Information Processing, Volume 255, Research and Practical Issues of Enterprise Information Systems II Volume 2, eds. L. Xu, Tjoa A., Chaudhry S. (Boston: Springer), pp. 887-891. 
integration with emphasis on connecting each enterprise difficultly obtained the real optimization of the whole. In Hu's [2] discourse, he proposed a theory of replacing the boundary of the enterprises with the boundary of the organizations from the point of view of management and economics. Based on the idea of on-demand business, IBM Corporation [3] points out that new business models would be developed along two paths: Enterprise Reconstruction and Industry Deconstruction. The core of enterprise reconstruction was to use Component Business Modeling (CBM) where IBM proposed to break down the business into component parts - each of which did not overlap and can be conveniently shared. Based on these theories, this article argues that the chief question of integrating the supply chain is to break down the supply chain as a whole and identify the business components after breaking the boundaries of the organizations.

On the other hand, Rayport et al [4] point out that every business today competes in two worlds: a physical world of resources that managers can see and touch and a virtual world made of information, and proposed the concept of virtual value chain. In a book about the value net Bovet [2] considers the modern supply chain as a digital value-adding chain that considers customers as its centre. Compared with supply chain managed ideology, value net emphasizes ideology that is digital, customeroriented, demand-driven, and systematic collaboration, and the definition of the dynamic net which had quick flows and was swift and adjustable. It advanced and applied up-to-date managed ideology and technology based on traditional supply chain ideology. In this sense, a competitive supply chain model should include at least three aspects - logistic flow, information flow and business flow.

Therefore, this article proposes a theory of virtual value net from an integral angle, and considers that capturing the unitary superiority of the supply chain must integrate the enterprises of the supply chain into the whole, divide the sections or organizations in the supply chain according to the customer-oriented business process of the supply chain after breaking the boundary of the enterprises, and research the association of the sections. Furthermore, the paper points out that a supply chain as a whole system should consist of three nets: logistics net, information resource net and organization net. And the integration strategy of the supply chain relates to how to construct and coordinate these nets and their nodes, so as to provide business value to the final customers, and be adaptable to business and technology changes.

The key factor in the virtual value net's construction is the selection of net nodes and the selection of net structure. This is to say that, based on sufficient analysis; we should clarify information resource from logistic flow in order to build an information resource net and logistics net. Then, we should apply SNA to evaluate the efficiency of both nets so that both nets could be dynamically regulated according to environment changes. The organization net should be kept consistent with the changes of these two nets and finally achieve optimization of business flow. 


\section{INTEGRATION EVALUATION MODEL}

Appropriate evaluation of the virtual value net is essential to the accomplishment of its optimization. This article specifies several critical evaluation indexes in order to achieve a complete and ideal net evaluation. The indexes are "customer value creation" and "nets value-adding capacity" (including logistic cost, nets' response time, and nets' degree of satisfaction).

Customer value creation is the core value in the virtual value net which means "the value that enterprises transfer to customers", i.e. the value of product or services provided by enterprises. The composition of customer value is illustrated in figure 1:

\section{The degree of demand satisfaction}

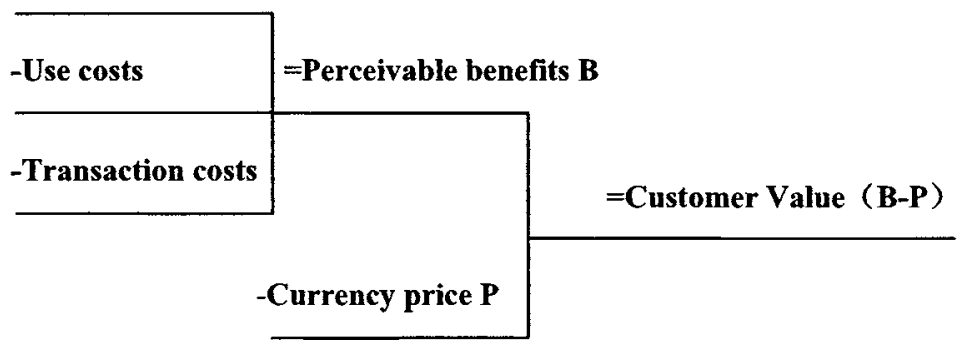

Figure 1. The Composition of Customer Value

Nets' value-adding capacity could be measured by three aspects: logistic cost [5], nets' response time [6]), and nets' degree of satisfaction [7].

In order to synthesize three aspects of net's value-adding capacity, we apply Analytic Hierarchy Process (AHP) [8], which is widely used in a multiple criteria decision making context to calculate the weight of each aspect. To measure attribute weights using AHP, subjects make a series of pairwise comparison judgments on a nine point ratio scale, $(1 / 9,1 / 7,1 / 5,1 / 31,3,5,7,9)$, the relative importance of the attributes with respect to the pertinent goal. The right eigenvector of the square matrix

of judgments is obtained and normalized to unity to obtain the vector of weights

$$
\sum_{i=1}^{n} W i=1
$$

Then, we synthesize the three indexes - logistic cost, nets' response time, and nets' degree of satisfaction:

$$
Y=\sum_{i=1}^{n} W_{i} Y_{i}
$$

Yi stands for index $i$; Wi stands for relevant weight of the index $i$.

In the light of the evaluation indexes and model above, we could figure out the effectiveness and efficiency of the entire supply chain through accurately calculating the quantitative indexes and properly judging the qualitative indexes. Based on this evaluation, we could constantly adjust the structure of three nets in order to ensure the quality of integration. 


\section{THE INTEGRATION OPTIMIZATION MODEL}

In the analysis of the virtual value net's capability, this article calculates the target value of two nets in virtue of the indexes of SNA e.g. nodal degree, density, and centrality and so on. Social network analysis (SNA) [9] is the quantitative research about social relations.

Here we use the Relative Centrality $C_{R D}^{\prime}$ to calculate a certain node's connection degree.

$C_{R D}^{\prime}(x)=$ (the 'input' nodal degree of $\mathrm{x}+$ the 'output' nodal degree of $\left.\mathrm{x}\right) /(2 \mathrm{n}$ 2),

Among which,

Sum of nodal degrees $=\sum_{i=l}^{g} d\left(n_{i}\right) \Rightarrow \sum_{I=S_{1}}^{S_{X}} d\left(n_{I}\right)+\sum_{l=S_{X+1}}^{G}\left[d\left(n_{l}\right) \times t\right]$,

$s_{1} s_{x}$ are not circular nodes,

$s_{X+1} \ldots G$ are circular nodes, $t$ amounts to the number of circulations

After calculating the centrality degree of every node, we could select the nodes of high centrality degree, then integrate periphery nodes into one node and finally form a high grading point.

A practical example of the application of the virtual value net is the design of the supply chain management model of the Luen Thai Corporation. Using theories of virtual value net, this example analyzes the core of the Luen Thai supply chain city model according to the construction method of the virtual value net, which proves the feasibility and efficiency of the model we proposed.

\section{CONCLUSIONS}

Integration of the supply chain is not only the business integration of the organizations in value-added chain/net, but also integration of the information resource net, the logistics organization net and organization net of the supply chain. The article combines the theory of the virtual value chain with the CBM of IBM, the boundaries of organization, SNA, and proposes the theory of a virtual value net in which the organizations' boundaries are regarded as transparent, activities in business processes are selected and organized as business components, logistics flow and information flow are analyzed and optimized according to the integration degrees of business components.

The continuous study could gradually introduce more indexes e.g. cost, quality, speed and so on, and include a more quantitative analysis for improving the whole net's efficiency. Moreover, the integrated strategy of the information resource net and the relative organization transformation can be analyzed further. 


\section{ACKNOWLEDGEMENTS}

This research was supported by the Peking University Luen Tai center for Supply Chain System R\&D.

\section{REFERENCES}

1. D. Li, Luen Thai: Creating world-class supply chain of clothing(2005). http://www.exam88.com/xueli/MBAMPA/zixun/200603/xueli_17923.html (Accessed July 1, 2007)

2. B. Hu, Logistics Organizations and Specialization Division (Economic Science Press: Beijing, 2003).

3. T.L. Saaty, The Analytic Hierarchy Process, McGraw Hill International. Volume 1, Number 3, pp.34-36, (1980).

4. D. Bovet, J. Martha, and R.K. Krame, Value Network (Post \& Telecom Press: Beijing, 2004).

5. H. Ye, Value Chain Analysis and Evaluation under Collaborative Product CommerceMode. Ph.D Thesis, Zhejiang Gongye University (2004).

6. J. Scott, Social network analysis (SAGE Press: New York, 1991).

7. J.F. Rayport and J.J. Sviokla, Exploiting the Virtual Value Chain, Harvard Business Review. Volume 12, Number 2, pp.73-76, (1995).

8. Y. Ding, Simulation Model for Calculating Response Time of Supply Chain Network, Joumal of System Simulation. Volume 2, Number 10, pp.2496-2499, (2005).

9. S. Zhou, Study on Performance Measurement System for Supply Chain Based on Coordination, Science-Technology and Management. Volume 3, Number 2, pp.39-419, (2005). 